The appearances were typical of Crohn's disease with discrete aphthoid or serpiginous ulcers with normal intervening mucosa. Only one patient showed the characteristic histological features, ${ }^{4}$ and a further six showed non-specific inflammatory changes.

Others.-This group comprised 26 patients who had strictures shown radiologically but who did not fall into any of the other categories. Eight patients were normal endoscopically, seven had muscle spasm only, two had carcinomas, three had benign adenomatous polyps in association with spasm, two had Crohn's disease and two had diverticular disease. Examinations failed in two patients.

\section{Discussion}

Strictures are often found that do not show radiological characteristics typical of one condition even though double contrast studies may provide helpful additional information and may indicate or exclude disease elsewhere in the large bowel. We have shown that colonoscopy provides a safe, reliable, and effective way of elucidating the differential diagnosis of strictures of the large bowel.

Of the 160 strictures examined 96 were in the signmoid colon but beyond the reach of the conventional rigid sigmoidoscope. This is a notoriously difficult area to examine radiologically and similarly has proved to be a difficult region to negotiate with the colonoscope, particularly in the presence of diverticular disease. One group of workers reportęd a $47 \%$ failure rate in the study of the differential diagnosis of carcinoma from diverticular disease. ${ }^{5}$ Our failure rate in this group was $8 \%$ and we believe that the difference may be due partly to the routine use of fluoroscopy and partly to slow and painstaking endoscopic technique in these patients.

Altogether $90 \%$ of the strictures examined were within reach of the medium length $(105-110 \mathrm{~cm})$ instruments, which are easier to handle, more durable, and less expensive to repair or replace; they should be used where possible. The two-channel "operating" colonoscope has, surprisingly, proved particularly easy to use in spite of its diameter.

If patients with longstanding ulcerative colitis develop strictures the colon is usually removed because it has until now been impossible to distinguish between malignant and benign strictures. With diagnostic colonoscopy surgery may be avoided in some patients. It is becoming apparent that the precancerous epithelial dysplasia (atypia) associated with the development of a carcinoma in longstanding ulcerative colitis $^{6}$ may be patchy and may spare the rectum, as in one of our cases. ${ }^{7}$ Nevertheless, it is almost invariably present in the mucosa immediately surrounding a carcinoma, ${ }^{8}$ and it is therefore imperative to take multiple biopsy specimens from any strictured area in patients with ulcerative colitis. Provided there is no evidence of epithelial dysplasia in these biopsies the stricture is almost certainly benign.

During colonoscopy it is difficult to make an accurate diagnosis on direct vision alone and biopsy specimens are essential. Malignant polyps often look benign, benign polyps malignant, and inflamed mucosa normal. ${ }^{9}$ In certain conditions, however, the converse may be true. In eight of our nine patients examined for Crohn's disease the biopsy specimens showed only nonspecific inflammatory changes in spite of the typical macroscopic appearance. This probably reflected the small, relatively superficial specimens which were obtained. We have found that the results of cytological and histological examinations of biopsy material correlate well and believe that taking biopsy specimens by brushing and washing is valuable in the colonoscopic diagnosis of strictures, particularly those through which it is impossible to pass the instrumeni.

The diagnosis was proved wrong in $52 \%$ of the 104 cases in which the clinicians and radiologists were prepared to make a probable diagnosis. In the remaining 50 patients a positive diagnosis was made on colonoscopy in all except two. Laparotomy was probably avoided in over half of this series as a direct result of colonoscopy, and though we have failed in 5\% of our patients this failure rate is small compared with the obvious clinical and economic benefits of the whole series.

\author{
References \\ 1 Woolf, W. I., Shinya, H., and Ozoktay, S. Z., American Fournal of Surgery, \\ $1972,123,180$ \\ 2 Williams, C. B., and Teague, R., Gut, 1973, 14, 990. \\ 3 Williams, C. B., et al., British fournal of Surgery, 1974, 61, 673. \\ ${ }^{4}$ Lockhart-Mummery, H. E., and Morson, B. C., Gut, 1964, 5, 493. \\ 5 Dean, A. C., and Newell, J. P., British fournal of Surgery, 1973, 60, 633. \\ ${ }^{6}$ Morson, B. C., and Pang, L. S. C., Gut, 1967, 8, 423. \\ 7 Evans, D. J., and Pollock, D. J., Gut, 1972, 13, 566. \\ ${ }^{8}$ Morson, B. C., personal communication, 1975. \\ ${ }^{9}$ Dilawari, J. B., et al., Gut, 1973, 14, 426.
}

\title{
Aspects of Sexual Medicine
}

\section{An Approach to the Gynaecological Patient}

\author{
PHILIP RHODES
}

British Medical fournal, 1975, 3, 361-363

\section{Privacy}

Doctors in general have often been upbraided for not having much insight or understanding of sexual disorders, and perhaps this criticism should be accepted. But it is not surprising that

Faculty of Medicine, University of Adelaide, Australia PHILIP RHODES, F.R.C.S., F.R.C.o.G., Dean there may have been reticence on the part of the medical profession, because there has not until recently been a reasonably received body of knowledge on sexual matters, nor was there suitable, convincing, and tested treatment for many of the sexual disorders. This is still largely true, though recently genuine attempts have been made to define and put on a scientific basis the diagnosis and management of sexual problems. It may be true that the doctors have been conservative, but they have been a useful brake on enthusiastic amateurs, of whom there are many in the sexual sphere, whose pet nostrums and theories have not had the all-embracing application that they fondly imagined. Sexual problems are very individual, affecting the 
whole personality, and arising out of it. Experienced doctors have therefore been slow to tamper with something which is so basic to human existence and where potentially they may do more harm than good. This is no area for superficial snap judgements.

Despite the plethora of articles and talk and discussion about sex in recent years it is still a matter of privacy. Few if any people can be objective about themselves or others, and probably all have their sexual prejudices. This realization must make the individual doctor analyse himself as far as he or she is able so that a decision may be reached on suitability of temperament to deal with sexual problems. In other subjects of medicine doctors do this and they recognize their unsuitability, for a variety of reasons, to practise in certain areas. The talents of different doctors are variable. Those without the aptitude for sex medicine should perhaps hand on their cases to others just as they would in other specialties. But obviously all doctors in clinical practice should try to recognize sexual disorders in their patients.

\section{Variety of Guises}

The recognition of a sexual problem is not always easy, since comparatively few patients will complain of such a disorder overtly. Indeed they may not even know that they have a problem and it may therefore masquerade under a variety of guises and psychosomatic symptoms, and these may be referable to any system, though in women they will often present as some dysfunction of the genitalia. Moreover, it is all too easy to refer any psychosomatic symptom to some upset in sexual function, for each of us probably has some sexual problem, and if the personality is probed deeply enough it can be unearthed and given undue prominence when the essence may lie elsewhere.

Even when there is a recognizable sexual problem it is not necessarily wise or helpful to the patient to try to do anything about it. Older patients in particular may have come to make an adjustment to their problems, and so may have achieved some degree of mental harmony which is best not disturbed for fear of awakening old ghosts which had been laid. A nicety of judgement is here often required. The present frank discussions of sex in the popular press, in journals, and in broadcasting often heighten tension in those who would otherwise not worry about their sexuality but who are made to think that they may be abnormal. Reassurance about the infinite variety of sexual behaviour and therefore of individual variability is often the best therapy, unless there is an obvious phobia about any particular form of sexual behaviour which has been triggered by publicity.

\section{Gynaecological Patients}

Masters and Johnson produced an elaborate form of history taking in those cases where there was a known sexual problem. ${ }^{1}$ This sort of history is very time-consuming and requires facilities far beyond the average capacity of the doctor or gynaecologist. It must be remembered that the Masters and Johnson clinic was specifically set up to deal with sexual problems and that it therefore attracted those couples who knew that they had them. These patients had reached such a pitch in their marital relationships that they were prepared to spend much time and money on treatment and submit themselves to a variety of unfamiliar sexual techniques as well as lay bare their sexual lives and fantasies. With these self-selected patients the elaborate history can, perhaps should, be undertaken so that a profile of the patients' sexual upbringing, beliefs, imaginings, dissatisfactions, satisfactions, irrationalities, fetishes, and practices may be built up and understood so as to form a basis for rational therapy.

As specialist clinics for sexual problems begin and become active, as they are already doing, family doctors and gynaecolo- gists will need to discover the people who have sexual difficulties and decide whether these patients need specialist help or whether they can be helped by the doctor with a general interest in sexual disorders. The advice offered below is at the preliminary level of definition of the individual's problem.

\section{NON-VERBAL SIGNALS}

Dress, cosmetics, hair style, and gait tell something of the image that a woman has of herself. The evidence must not be pressed too far, for a nymphomaniac might be hiding beneath the most dowdy exterior. But wittingly or unwittingly these non-verbal signals are being sent out and received by the doctor and may give helpful clues, though they have to be judged in the light of the fashion of the day and the cult to which the patient is likely to belong.

The socio-economic class is to some extent a determinant of sexual behaviour. There is likely to have been more reading about sex in social classes I and II than in the others, and also likely to have been more experimenting sexually in matters such as fellatio and cunnilingus, whereas these may be inhibited in the lower social classes by their culture and the received attitudes of correct behaviour as between men and women. Fewer members of social classes IV and V will have adequate knowledge of contraception, so that they may have more anxieties about sexual congress and yet be relatively more unable to express them.

Age is not necessarily a guide to sexual attitudes or prowess, but again there will be general differences between the young and the old, and between those who are married and those who are single, and those who have children and those who do not, and between those who are engaged and those who either wish to have or do not wish yet for a stable relationship. The permutations are endless and sexual attitudes and problems must have their bases in body type, education, upbringing, family, school, friends, press, broadcasting, advertising, sexual opportunities and experiences, and knowledge of contraception. In the individual an attempt must be made to give these due weight in deciding about the importance of a sexual problem. The terms of the equation are almost impossible to control. But all these factors must in some measure be determinants of the type of advice ultimately given.

\section{HISTORY}

Disordered periods are usually due to disturbed endocrine function or uterine abnormality and these must be investigated in the usual fashion. But the endocrine abnormality may well be due to psychic disturbance and this may need investigation, though not all upsets in psychological functioning are sexual. There may be financial, housing, or other worries, and among these may be fear of infertility or of childbearing. Dysmenorrhoea too may or may not have a sexual basis. Discharges from the vagina are often of infective origin, some of them venereal, and previous sexual behaviour known to the patient may worry her greatly. The more physiological discharges from the cervix and Bartholin's glands under sexual stimulation may also cause anxiety. Pruritus vulvae, after the usual causes have been excluded, may have sexual connotations. The obstetric history, the numbers of children, of stillbirths, of neonatal and infant deaths, and of miscarriages are obvious potential factors in sexual attitudes. And there is the ever present anxiety about infertility, which it is now widely known may be due to gonorrhoea and previous abortion.

In taking a sexual history (see Appendix), the part to do with sexual intercourse, the doctor may well in the first instance inquire about any pain there may be during or after the act. This is a question expected by the patient and therefore is a useful lead into the further history. The response to it may determine whether it is best to pursue the matter later in the interview and examination, or whether it will be possible to 
continue on a similar tack. There may be other difficulties, especially lack of desire or dryness of the vagina, and these may be elicited in reply to a question about any other difficulties or problems with intercourse. Further detail is often best left until one is quite sure that there is no obvious gynaecological disease, though the doctor must at some point ask about previous venereal disease and about how many sexual partners there have been. This may give the clue to whether investigation is needed for asymptomatic venereal disease, and especially for investigation for gonorrhoea, which is now an epidemic disease.

\section{EXAMINATION}

The gynaecological examination proceeds along the usual lines, with a glance for acne and hirsutism, which can be distressing to some patients. The breasts are always inspected and palpated, and smallness or largeness or other abnormality may be of significance. The response to the vaginal examination is worthy of note. It may range from vaginismus, making examination impossible, to such total relaxation that the pelvis may be felt as completely as under general anaesthesia. Both of these extremes need further investigation in the sexual history, for the first implies an anxiety neurosis and the second an almost hysterical anaesthesia, though it must be remembered that physiotherapists and ballet dancers may have such superb muscular control that they can override their usual mental reactions to examination. The majority of women will, of course, have reactions intermediate between these extremes, and this occasions more difficulty in deciding about their significance. Where relevant, further inquiry centres on relationships with the husband or sexual partner(s), and different reactions with different men, with the frequency of intercourse, which varies especially with age, and with the frequency of orgasm, which is very variable and does not necessarily imply dissatisfaction.

\section{Life Style}

It is impossible to cover all the nuances of the sexual history in a short space. The key to the treatment of sexual disorders lies in no specific system of therapy or drug, except very rarely. The disorder is the outcome of the total personality and attitudes, and though there are methods of dealing especially with the sexual problem real management demands some alteration in the life style of the patient. It is this style which is being investigated in the sexual history, and explains why it is almost impossible to pin down specific syndromes.

Simplistic diagnostic labels are not the aim in sex medicine. Instead one is groping for the fullest possible understanding of the patient, so that there may be a reasonable basis on which to offer advice. And sometimes it is better to offer no advice at all, especially if the patient would find gratuitous help intolerable. Even when it is decided that help is needed the advice has to be carefully tailored to suit the individuals concerned, for, despite the high talk of sexual emancipation in general, not all individuals are capable of or willing to indulge in the various sexual therapies now available.

\section{Appendix}

AN OUTLINE GUIDE FOR A SEXUAL HISTORY IN WOMEN

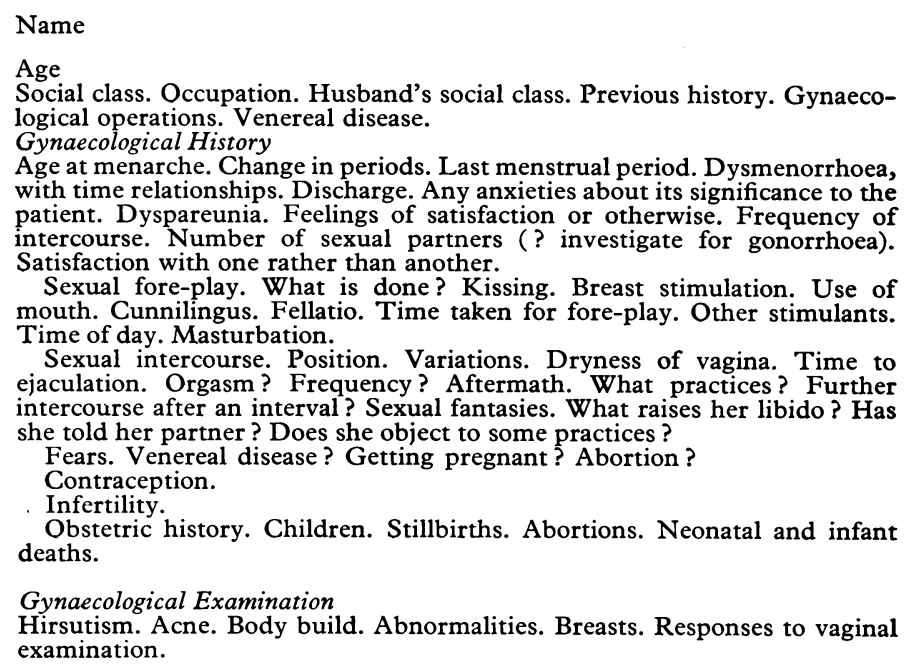

Age at menarche. Change in periods. Last menstrual period. Dysmenorrhoea, with time relationships. Discharge. Any anxieties about its significance to the patient. Dyspareunia. Feelings of satisfaction or otherwise. Frequency of intercourse. Number of sexual partners (? investigate for gonorrhoea). intercourse. Number of sexual partners

Sexual fore-play. What is done? Kissing. Breast stimulation. Use of Sexual fore-play. What is done? Kissing. Breast stimulation. Use of
mouth. Cunnilingus. Fellatio. Time taken for fore-play. Other stimulants. Time of day. Masturbation.

Sexual intercourse. Position. Variations. Dryness of vagina. Time to ejaculation. Orgasm? Frequency? Aftermath. What practices? Further intercourse after an interval? Sexual fantasies. What raises her libido? Has she told her partner? Does she object to some practices?

Fears. Venereal disease? Getting pregnant ? Abortion?

Contraception.

Infertility.

Obstetric history. Children. Stillbirths. Abortions. Neonatal and infant deaths.

Gynaecological Examination

Hirsutism. Acne. Body build. Abnormalities. Breasts. Responses to vaginal examination.

\section{Reference}

${ }^{1}$ Masters, W. H., and Johnson, V. E., Human Sexual Inadequacy. London and Edinburgh, Churchill, 1970.

\section{Any Questions?}

We publish below a selection of questions and answers of general interest

\section{Psittacosis in Children}

\section{How common is psittacosis in children and how should it be treated?}

Psittacosis is acquired through household or occupational exposure to birds infected with Chlamydia psittaci. Healthy birds that may have latent infection acquired as fledglings usually do not spread the disease. But if such birds, especially psittacines, become sick owing to stress-from overcrowding or underfeeding during transport or in pet shops-they may shed many of the organisms. Human infection may occur at any age but is commonest between 20 and 50 years. The fatality rate, if untreated, approaches $20 \%$. Severely ill patients should be treated with intravenous tetracyclines $(0.5 \mathrm{~g}$ every 12 hours for adults), and mild cases with oral tetracyclines (0.25-0.5 g 6-hourly). Oral treatment should be continued for one to two weeks after the patient becomes afebrile. Erythromycin and doxycycline are effective alternatives. In all cases the diagnosis should be confirmed by complement fixation tests, but, as the antibody is slow to appear, treatment in epidemiologically suspicious cases should not wait for laboratory confirmation.

\section{Immunization against Japanese Encephalitis}

What is the current position about immunization against fapanese encephalitis?

Formalin-inactivated Nakayama strain Japanese B virus vaccine has been widely used since it was first developed and studied by Sabin et al. in 1943. ${ }^{1}$ The virus is grown in suckling mouse brain and may be contaminated with residual amounts of myelin, which potentially could give rise to demyelinating encephalitis. Virus growth is substantially better in suckling 